\title{
Affinités sociales, intermédiations politiques
}

\author{
Lorenzo Barrault-Stella (CRESPPA-CSU), Patrick Lehingue (CURAPP-ESS)
}

La rationalisation historique des modes de domination a conduit dans de nombreux pays à l'émergence d'un champ politique relativement autonome, induisant une division entre quelques professionnels et une immense majorité de «profanes ${ }^{1}$. En France, l'accélération de la professionnalisation ${ }^{2}$ comme le renforcement de la sur-sélection sociale des acteurs politiques ces dernières décennies ont sans doute accentué la dynamique d'autonomisation du champ politique. Pourtant, comme pour d'autres champs de production de biens symboliques, l'étude des mondes politiques ne peut faire l'économie d'une analyse des sanctions externes rituellement organisées par le biais d'élections visant à renouveler et, plus encore, à recréditer les représentants comme, au-delà, les mécanismes de délégation. Aussi, les événements électoraux concourent à l'actualisation du champ politique et chaque échéance procure l'occasion d'examiner les modalités d'agencement entre espace social et espace politique. Dans les années 1960-1970 en France, des homologies structurales entre l'espace des prises de position politiques et l'espace social ont pu être statistiquement validées ${ }^{3}$, pour être ensuite fortement nuancées ${ }^{4}$. Elles sont remises à l'ordre du jour à l'occasion du referendum de 2005 sur le traité européen, tant variables sociales et préférences politiques résonnent alors particulièrement bien ${ }^{5}$. L'affaire paraît entendue : l'homologie structurale joue inégalement d'un scrutin à l'autre, selon l'offre politique du moment, le degré de saillance des enjeux débattus et le quantum d'effervescence des campagnes. Expliquer cette variabilité nécessite toutefois de questionner à nouveaux frais les mécanismes de (dés)ajustement entre «l'offre politique » et les « attentes sociales », et notamment le système d'intermédiation ${ }^{6}$ entre champ politique et espace social en période électorale. Car les activités politiques restent périodiquement dépendantes de leurs réceptions dans les différentes régions composant l'espace social. Aborder les pratiques électorales sous l'angle des intermédiations comme le propose ce dossier permet de prolonger les analyses dites contextuelles du vote, en inscrivant le questionnement dans une sociologie relationnelle des transformations des rapports au champ politique et donc des modes de domination.

\section{Restituer le vote au plus près de ses conditions sociales de production}

\footnotetext{
${ }^{1}$ Pierre Bourdieu, «La représentation politique. Eléments pour une théorie du champ politique », Actes de la Recherche en Sciences Sociales, $\mathrm{n}^{\circ} 36 / 37,1981, \mathrm{p} .3-24$.

2 Julien Boelaert, Sébastien Michon, Étienne Ollion, Métier : député. Enquête sur la professionnalisation de la politique en France, Raisons d'agir, Paris, 2017.

${ }^{3}$ Daniel Gaxie, «Les logiques du recrutement politique », Revue française de science politique, vol. $30, \mathrm{n}^{\circ} 1$, 1980, p. 5-45.

${ }^{4}$ Bruno Cautrès, Flora Chanvril, Nonna Mayer, «Retour sur l'hypothèse de «l'homologie structurale »: les déplacements des catégories sociales dans l'espace politique français depuis La Distinction », in Philippe Coulangeon, Julien Duval (dir.), Trente ans après La Distinction de Pierre Bourdieu, Paris, La Découverte, p. 327-337.

5 Patrick Lehingue, «Le Non français au traité constitutionnel européen (mai 2005). Sur deux lectures « polaires » du scrutin », Actes de la recherche en sciences sociales, $\mathrm{n}^{\circ}$ 166-167, 2007, p. 123-139.

${ }^{6}$ Olivier Roueff, «Les homologies structurales : une magie sociale sans magicien ? La place des intermédiaires dans la fabrique des valeurs », in Philippe Coulangeon, Julien Duval (dir.), Trente ans après La Distinction de Pierre Bourdieu, Paris, La Découverte, 2013, p. 153-164.
} 
Les articles réunis dans ce dossier s'attachent en ce sens à saisir de manière contextualisée les affinités éventuelles entre espace politique et espace social en éclairant les diverses médiations qui mettent (ou non) en relation l'offre politique et les électeurs de différents groupes sociaux. Autrefois entretenue dans les manuels d'instruction civique mais toujours présente à l'état latent lorsqu'il s'agit de signifier ce que voter veut dire, la mythologie démocratique voudrait que le vote soit une pratique individuelle, élaborée et patiemment murie par chacun en son for intérieur, dans un dialogue intime et secret où se confronteraient des considérations personnelles. Les technologies électorales, au premier rang desquelles l'isoloir historiquement nommé « cabine d'isolement » ${ }^{7}$, matérialisent et confortent cette vision atomisée. Celle-ci va le plus souvent de pair avec le désencastrement biographique des pratiques de vote qu'on ne cesse, sous des formes toujours renouvelées, d'analyser comme étant placées en état d'apesanteur social, comme délivrées de « la camisole de force des déterminants sociaux $\gg$ qui les enserraient ${ }^{8}$.

On sait depuis longtemps qu'il n'en est rien. Des enquêtes empiriques pionnières ont par exemple établi que «les gens qui travaillent ou vivent ou se distraient ensemble sont enclins à voter pour les mêmes candidats » ou encore, de manière plus caustique que « les vrais électeurs hésitants - les citoyens à l'esprit ouvert (open minded) qui font de sincères efforts pour soupeser les enjeux et les candidats sans passion et pour le bien de leur pays dans son ensemble - existent principalement dans la propagande de campagne, dans les livres d'éducation civique, dans les films, ou dans la pensée d'idéalistes politiques. Dans la vraie vie, ils sont réellement peu nombreux ${ }^{9}$. Empiriquement bien établies malgré quelques scories tenant à leur caractère pionnier, ces recherches n'ont depuis cessé d'être au mieux ignorées parce que considérées comme triviales, au pire contestées au seul motif qu'elles relèveraient d'un passé heureusement dépassé. En effet, l'action volontariste des entreprises politiques sur les marchés électoraux (monopole de distribution des investitures, délimitation de l'univers du pensable, activation des identités partisanes, etc.) et la sophistication croissante du travail de rationalisation présidant à la conquête des suffrages (à l'instar de logiciels d'identification des électeurs précédant les pratiques de porte-à-porte) semblaient jusqu'à récemment déclasser les médiations non organisationnelles et pas toujours stratégiquement orientées qui s'établissaient entre l'offre politique et les électeurs. Acte de décès sans doute prématuré ! Les «désalignements » électoraux, la croissance de la défiance affichée à l'égard des institutions politiques, le déclin du nombre de participants au rituel électoral, la perte d'emprise des partis et de leurs réseaux dont témoignent des mouvements comme ceux d'Occupy Wall Street, des Indignados, ou des Gilets Jaunes, ou encore les échecs récurrents des «spin doctors » et autres haruspices du marketing électoral, redonnent toute leur valeur aux groupes primaires d'appartenance des citoyens et aux sociabilités « ordinaires ».

\footnotetext{
${ }^{7}$ Alain Garrigou, « Le secret de l'isoloir », Actes de la recherche en sciences sociales, $\mathrm{n}^{\circ}$ 71-72, 1988, p. 22-45.

${ }^{8}$ Victor O. Key, The Responsible Electorate, Harvard University Press, 1966, p. 8.

${ }^{9}$ Paul Lazarsfeld, Bernard Berelson, Hazel Gaudet, The People's Choice. How the voter makes up his mind in a presidential campaign, New York, Columbia University Press, 3ème edition 1968 [1944], p. 27.
} 
Puisque «cela fait plusieurs décennies que la science du vote n'est plus, majoritairement, une sociologie ${ }^{10}$, suivre ces pistes implique de faire au moins deux pas de côté par rapport à de nombreux travaux d'analyse électorale. D'une part, rompre avec le penchant intellectualiste qui conduit à survaloriser l'acte de vote pour les citoyens, alors que les sciences sociales ont établi que l'attention portée aux enjeux du champ politique est en moyenne assez faible et surtout très inégalement répartie socialement ${ }^{11}$. Et d'autre part, ne pas s'en tenir aux seuls sondages atomistiques et à quelques variables d'identification sociale (la fameuse dichotomie CSP + ou - par exemple) pour étudier les comportements électoraux. Dans le souci de gagner en réalisme sociologique ${ }^{12}$, il s'agit donc de réinscrire les électeurs dans « leurs contextes » de vie quotidienne et, plus précisément dans le cadre de ce dossier, de restituer la variabilité des médiations avec le champ politique qu'impliquent leurs divers ancrages territoriaux et sociaux.

\section{De la mobilisation à l'intermédiation électorale}

Les articles réunis dans ce dossier participent en ce sens au renouvellement des analyses électorales contextuelles par leur souci commun d'objectiver les mécanismes de production des votes au sein de différents milieux sociaux et de différents territoires (inégalement urbanisés) au prisme des intermédiations avec le champ politique ${ }^{13}$. En science politique, la question de l'intermédiation est notamment abordée par les nombreux travaux sur les campagnes électorales, qui montrent comment les organisations politiques tentent de mobiliser. Depuis les études pionnières de Paul Lazarsfeld et de son équipe dans les années 1940, on ne compte plus les recherches sur le travail de mobilisation politique en conjoncture électorale $^{14}$. Ce travail politique s'effectue presque toujours dans une dynamique de concurrence entre intermédiaires. Mais cette concurrence est généralement pensée comme mettant aux prises les seules entreprises politiques, voire leurs relais et des acteurs à la lisière du champ. La sociologie électorale décrit ce travail comme des «dispositifs politiques de mobilisation », en complément des «dispositifs administratifs de "facilitation" du vote ${ }^{15}$. Si l'analyse de ce type d'intermédiation est précieuse, de nombreux travaux soulignent la rétraction des réseaux partisans de médiations électorales ${ }^{16}$. À se focaliser sur les seules

\footnotetext{
${ }^{10}$ Céline Braconnier, Une autre sociologie du vote. Les électeurs dans leurs contextes, Paris, Lejep-Lextenso éditions, 2010, p. 17.

${ }^{11}$ Daniel Gaxie, Le cens caché. Inégalités culturelles et ségrégation politique, Paris, Seuil, 1978.

${ }^{12}$ Céline Braconnier, Jean-Yves Dormagen, La démocratie de l'abstention, Paris, Gallimard, 2007.

${ }^{13}$ L'articulation des dispositions et des diverses intermédiations susceptibles de politiser (au-delà du seul vote) les groupes sociaux est au cœur du programme de recherche proposé par Lorenzo Barrault-Stella et Bernard Pudal à partir d'une relecture des travaux de Pierre Bourdieu sur ces questions : « Retour sur la politisation des classes populaires. Propositions pour une analyse des états de matière du politique et de leur convertibilité », in La politique désenchantée? Perspectives sociologiques autour des travaux de Daniel Gaxie, Rennes, PUR, 2019, p. 105-128.

${ }_{15}^{14}$ Par exemple Clément Desrumeaux, Rémi Lefebvre (dir.), « Faire campagne », Politix, n $113,2016$.

${ }^{15}$ Ces questions sont aujourd'hui fortement intriquées à des enjeux de gouvernement du vote que l'on retrouve au niveau international concernant l'encadrement électoral des classes populaires : Hélène Combes, Gabriel Vommaro, «Gouverner le vote des «pauvres ». Champs experts et circulations de normes en Amérique latine », Actes de la recherche en sciences sociales, ${ }^{\circ} 216-217,2017$, p. 4-23.

${ }^{16}$ Par exemple Sandrine Lévêque, Anne-France Taiclet (dir.), À la conquête des villes, Villeneuve d'Ascq, Presses Universitaires du Septentrion, 2018.
} 
médiations issues du champ politique, on serait tenté de conclure à la généralisation d'un processus de désintermédiation $^{17}$.

Toutefois, la sociologie des intermédiaires gagne à être élargie au-delà des institutions politiquement différenciées, de leurs réseaux et du travail de campagne médiatiquement valorisé. Les articles réunis ici l'attestent : une partie du système d'intermédiation électorale est invisibilisée du fait de son caractère apparemment moins formel, moins officiel et moins «légitime». C'est le cas de toutes les médiations non déployées spécifiquement en conjoncture électorale et qui empruntent des formes officieuses, «anodines », « ordinaires » parce qu'elles s'appuient sur des structures sociales routinières, à l'image des relations au sein des groupes d'appartenance, qu'il s'agisse de la famille, des amis, des collègues ${ }^{18}$, etc. Outre de potentielles conversations portant explicitement sur les élections au sein de ces collectifs, ils façonnent tacitement les rapports aux biens politiques comme à leurs modes de consommation. Comme l'avait souligné l'école de Columbia, les médiations électorales en jeu dans ces groupes primaires se repèrent aux usages toujours sélectifs des médias et aux appropriations obliques que leur font subir des agents sociaux moins passifs ou vulnérables qu'on ne se plaît parfois à les imaginer ${ }^{19}$. Cette tendance à l'oubli des intermédiaires les moins pensés politiquement et les moins visibles peut être rapprochée d'un double penchant légitimiste et stratégique dominant dans les analyses électorales, qui conduit à porter l'accent sur le travail politique officiel et les activités de rationalisation des entreprises de captation des suffrages.

Construire l'objet électoral sous l'angle des intermédiations avec le politique comme le propose ce dossier permet de décaler le regard par rapport à ces perspectives, en ne prenant pas seulement en compte les activités de mobilisation depuis le champ pour y ajouter l'observation des médiations émanant des groupes sociaux vers le champ. Sur ce point, la sociologie des biens culturels a insisté sur les luttes concurrentielles entre les intermédiaires et, en particulier, sur les dimensions intentionnellement stratégiques de l'intermédiation. Mais on touche ici à une limite de l'analogie entre culture et politique : les biens politiques ont la spécificité de faire l'objet d'une injonction généralisée à l'intérêt (via notamment la mythologie du citoyen éclairé $)^{20}$ et les produits électoraux se caractérisent par d'incessants rappels à la norme participationniste telle qu'elle a pu s'imposer historiquement. Cette singularité implique que la mise en relation entre l'offre politique et les électeurs des différents groupes sociaux puisse emprunter des voies particulièrement diversifiées et s'appuyer, non seulement sur des intermédiaires spécialisés à des degrés variés (tels les candidats, des militants, des relais médiatiques), mais aussi sur des intermédiaires non

\footnotetext{
${ }^{17}$ L'hypothèse est présente dans Rémi Lefebvre, « Les Gilets jaunes et les exigences de la représentation politique », La Vie des idées, 10 septembre 2019.

${ }^{18}$ L'entrée empirique par la scène résidentielle adoptée par plusieurs des articles du dossier ne doit pas conduire à écarter d'autres intermédiations susceptibles de structurer les rapports au champ politique, par exemple sur la scène professionnelle.

${ }_{19}$ «Les intellectuels ont toujours peine à croire aux défenses, c'est-à-dire à la liberté des autres, puisqu'ils s'attribuent volontiers le monopole professionnel de la liberté d'esprit (...) ils ont toujours besoin de mystifiés pour s'apparaître comme démystificateurs»: Pierre Bourdieu, Jean Claude Passeron, «Sociologues des mythologies et mythologies des sociologues », Les Temps Modernes, n²11, 1963, p. 1010.

${ }_{20}$ La consommation d'autres biens culturels ne fait pas l'objet d'injonctions aussi généralisées et de culpabilisation pour ceux qui s'abstiennent de consommer.
} 
spécialisés, non institués depuis le champ politique et pas toujours orientés stratégiquement vers la conquête de suffrages. Parmi ces derniers, on peut distinguer toute une gamme allant des plus visibles (dont certains, comme les courtiers électoraux et autres brokers ${ }^{21}$, ont déjà été identifiés par la littérature sur le clientélisme et les réseaux des organisations partisanes ${ }^{22}$ ) aux plus informels, ces derniers épousant les contours des groupes d'appartenance par entretien des sociabilités (familiales, amicales, professionnelles, etc.) et activation des normes qui structurent ces collectifs. Ainsi, par contraste avec les travaux sur les entrepreneurs ou les courtiers, ce n'est pas seulement l'intention des acteurs qui les fait accéder au statut d'intermédiaire politique, mais leur activité objective de mise en relation, après filtrage et décodage, des biens politiques et des groupes sociaux. D'ailleurs, ces différents types d'intermédiation avec le champ politique (spécialisés ou non, visibles ou informels) pourraient, par analogie, se retrouver dans les relations qu'entretiennent les groupes sociaux à d'autres espaces de production de biens symboliques.

\section{Une analyse relationnelle des diverses intermédiations politiques}

Ce décalage du regard permet de saisir la variabilité du poids relatif de chacune des formes d'intermédiation. Variabilité dans le temps avec, dans le cas de la France au moins, un recul des intermédiations spécialisées comme celles partisanes. Et variabilité dans l'espace, l'intensité comme les modalités du travail d'intermédiation variant fortement selon les territoires et les milieux sociaux qui les peuplent comme en attestent les articles du dossier. Ces contributions montrent de manière convergente la centralité des affinités sociales et des intermédiaires informels, inscrits dans les rapports sociaux du quotidien, qui peuvent façonner les goûts comme les dégoûts politiques tout autant voire davantage que le travail partisan, médiatique et militant. Les enquêtes soulignent que c'est en famille, entre amis, entre collègues, au travers de fugaces interactions quotidiennes souvent labellisées comme non politiques que s'interprète et prend (ou non...) sens l'offre électorale et que s'opèrent, le cas échéant, des arbitrages électoraux qui n'ont dès lors plus rien d'individuels ni même de secrets $^{23}$. Qu'il s'agisse de quartiers parisiens, d'une ville moyenne du Sud de la France ou encore d'un territoire rural, les rapports sociaux ne sont en aucun cas suspendus lors des conjonctures électorales. Celles-ci ne sont que des moments - parmi d'autres et inégalement sacralisés - où se (re)jouent et s'actualisent les relations entre les groupes. La recherche menée dans un arrondissement du Nord de Paris montre qu'une part importante des intermédiations électorales se révèle symbolique, passant subrepticement par les manières dont les électeurs se pensent et se représentent les autres groupes sociaux. En rappelant ce qui se passe en période électorale dans les groupes primaires comme la famille et les réseaux amicaux, les articles rejoignent les travaux anthropologiques sur le vote clientélaire appréhendées à travers les relations personnalisées qui peuvent se nouer entre les familles et

\footnotetext{
${ }^{21}$ Par exemple Herbert Kitschelt, Steven Wilkinson (eds), Patrons, Clients, and Policies. New York, Cambridge University Press, 2007.

${ }^{22}$ Frédéric Sawicki, Les réseaux du Parti socialiste. Sociologie d'un milieu partisan, Paris, Belin, 1997.

${ }^{23}$ Une piste qui reste à explorer concerne les individus isolés qui ne sont attachés à aucun groupe primaire : peuton faire l'hypothèse que ces derniers sont davantage tributaires d'intermédiations électorales « à distance », par la (seule ?) réception d'informations médiatiques ?
} 
dont la politique est partie prenante ${ }^{24}$. Ils analysent à nouveaux frais les dynamiques d'échanges interpersonnels dans la formation des préférences politiques, comme la contribution d'une association de pêche en Mayenne à la légitimation du Front National (FN) dans les classes populaires. Ils soulignent les logiques de la perte de croyance dans la valeur de l'institution électorale dans certaines régions intermédiaires de l'espace social, à l'instar des employés observés dans le Var.

Prendre en compte de la sorte les diverses appartenances et les entourages dans l'étude des déterminations du vote n'est évidemment pas une idée nouvelle. Outre les travaux de l'école de Columbia déjà cités, le renouveau contextualiste des analyses électorales aux ÉtatsUnis et en Grande-Bretagne depuis les années 1980 s'inscrivait en rupture avec une conception purement internaliste des préférences électorales ${ }^{25}$. Il pointait l'importance d'une sociologie des réseaux ${ }^{26}$, même si c'est parfois au risque de négliger le social incorporé sous la forme de dispositions relativement stables rendant non seulement possibles certaines interactions mais surtout façonnant leur déroulement comme leurs effets. En France, les analyses contextuelles menées depuis une quinzaine d'années ${ }^{27}$ ont de même souligné le poids des «dispositifs informels de mobilisation» pour désigner le rôle joué par les groupes primaires dans l'incitation à se rendre aux urnes, voire - mais plus rarement - dans l'orientation des votes.

En complément de ces travaux, la construction d'objet en termes d'intermédiation au cœur de ce dossier permet d'étudier la domination politique sous l'angle des rapports inégaux au champ politique. Ce qui conduit à porter le regard sur les diverses médiations à l'œuvre en conjoncture électorale entre le champ et ceux qui en sont - variablement du fait de la différenciation sociale et territoriale des intermédiations comme en témoignent les articles tenus à distance. La mise en relation au sein du dossier d'enquêtes contrastées suggère en la matière quelques régularités au-delà des variations contextuelles. D'une part, pour la période récente et quels que soient les sites observés, les médiations des organisations politiques voire la simple présence physique de militants tractant sur les marchés apparaissent rarissimes, notamment (mais pas seulement) pour les adhérents du FN pourtant étudiés dans ce dossier dans deux de leurs fiefs électoraux. La portée sociale des intermédiations spécialisées se révèle aujourd'hui des plus restreintes et la distance au champ politique - contrainte ou voulue - demeure ainsi, même en période électorale, le rapport modal au politique. Si elle est plus fréquente chez les employés et les professions intermédiaires d'une ville moyenne de la région PACA, cette posture se rencontre aussi - mais pour bien d'autres raisons et sous

\footnotetext{
${ }^{24}$ Gérard Lenclud, «S'attacher. Le régime traditionnel de la protection en Corse », Terrain, n²1, 1993, p. 8196.

${ }^{25}$ Alan S. Zuckerman (ed.), The Social Logic of Politics. Personal Networks as Contexts for Political Behavior, Philadelphia, Temple University Press, 2005.

${ }^{26}$ La perspective proposée par Robert R. Huckfeldt et John D. Sprague vise à élaborer une «théorie des réseaux d'influence politique » par des modélisations multivariées à partir d'échantillons probabilistes : «Discussant Effects on Vote Choice. Intimacy, Structure, and Interdependence », The Journal of Politics, 53(1), 1991, p. 122158. Mais leur travail tend paradoxalement à réduire le contexte au «réseau » et à déréaliser les rapports sociaux.

${ }^{27}$ Pour une discussion quant à l'articulation de ces travaux contextuels français avec ceux menés aux États-Unis et en Grande-Bretagne, voir la première partie de Julien Audemard, « De quoi le contexte est-il le nom ? Critique de l'usage de la notion de contexte en sociologie électorale », Revue française de science politique, vol. $67, \mathrm{n}^{\circ} 2$, 2017, p. 271-289.
} 
d'autres modalités - chez les résidents du 16ème arrondissement parisien parmi les plus fortunés. D'autre part, les classes populaires sont invariablement celles dont les intérêts sont les plus improbablement représentés en politique du fait de chaînes d'intermédiations particulièrement longues et entremêlées, en lien avec la très grande variabilité des formes d'identification aujourd'hui observables dans ces milieux. L'enquête dans un territoire rural montre par exemple combien certaines fractions populaires se tiennent à distance du rite électoral tandis que d'autres, parmi les plus stables et masculines, tendent à soutenir le FN, non du fait du travail partisan, mais plutôt par la médiation légitimante de son offre par des travailleurs indépendants. Il en va de même, en l'absence d'encadrement organisationnel, des processus d'identification symboliques générés par des tensions ethno-raciales au sein d'un quartier très populaire du 18ème arrondissement de Paris. Et les recherches rassemblées attestent que cet état des rapports populaires au champ politique contraste fortement avec les postures des diverses fractions des classes supérieures qui demeurent fortement attachées à la défense de leurs intérêts sociaux par le vote.

\section{Vote et ordre social}

Dès lors, les articles réunis dans ce dossier convergent par leur souci d'articuler pratiques de vote et ordre social. Rarement guidé par des professionnels de la politique dont on se défie souvent, mais opérant par des pratiques d'interconnaissance, de prise en compte du jugement des autres, d'échanges complices de goûts et de dégoûts, le travail d'ajustement et d'affinités entre offres, attentes et (éventuellement) soutiens électoraux réalisé par les intermédiaires apparaît très largement tributaire des diverses asymétries sociales structurant chacun des territoires étudiés. Ces intermédiations sont dans tous les cas incompréhensibles sans caractérisation sociale de ces contextes tant les affinités électorales se construisent en écho aux affinités électives des groupes (sociaux, ethno-raciaux, générationnels, etc.) d'appartenance. Leur analyse participe donc de l'étude des conditions sociales d'activation, d'actualisation et/ou de mise en sommeil de dispositions susceptibles de générer et d'orienter des pratiques politiques. Quels que soient les milieux et les territoires, les contributions suggèrent que c'est toujours dans la combinaison des dispositions sociales, des diverses intermédiations et de l'offre de représentation (qui délimite l'espace des possibles lors de chaque scrutin) que l'on peut expliquer les pratiques politiques. Les déterminations sociologiques restent donc centrales pour peu que l'on se donne les moyens empiriques de les observer, sans les réduire aux éventuelles corrélations entre une PCS et un vote. Sur ce point, certains articles abordent la question de l'articulation des différents rapports sociaux - de classes, de race, de genre ou encore de générations - dans l'étude des comportements politiques. Car du point de vue des médiations au sein des groupes d'appartenance, les modalités d'identification apparaissent en pleine transformation, à l'instar de l'affaissement tendanciel de la conscience de classe dans les milieux populaires, de la centralité des identifications ethno-raciales dans certains groupes ou encore du renouveau générationnel de petites différences distinctives au sein des classes supérieures.

Aussi, ces recherches de sociologie politique s'inscrivent pleinement dans les perspectives de l'analyse localisée des classes sociales, qui consiste à les saisir dans leurs 
relations et au concret ${ }^{28}$. Tel est le cas de l'enquête dans les «beaux quartiers » qui restitue la diversité des appréhensions du vote dans les fractions économiques des classes supérieures tout autant que les clivages générationnels qui peuvent s'y faire jour. L'étude menée au sein d'une ville moyenne du Var souligne aussi combien les rapports au vote sont indexés à des enjeux statutaires et aux relations entre les différents groupes. De même, lorsque les auteurs de l'article sur le 18ème arrondissement parisien insistent sur l'altérisation raciale comme clef de lecture centrale des élections au sein des milieux populaires, ils questionnent la singularité des rapports sociaux localisés et approfondissent l'hypothèse d'une socialisation politique résidentielle. Ces travaux invitent à accepter qu'une même «variable » puisse avoir des implications et des significations politiques différentes selon les espaces sociaux localisés ${ }^{29}$. Suivre cette piste ne dispense pas d'articuler l'analyse localisée à des recherches statistiques de plus grandes échelles. Plus que des oppositions entre échelles ou entre méthodes, il s'agit de regards complémentaires pour contribuer à l'analyse des composantes politiques des styles de vie des différentes classes sociales. Et des recherches récentes, quantitatives comme qualitatives, ont rappelé avec force combien les goûts politiques étaient intriqués aux morales de classes et aux visions du monde des différents groupes sociaux ${ }^{30}$. En montrant comment les diverses inégalités se réfractent dans l'ordre politique, par le biais des pratiques électorales et des intermédiations qu'elles supposent, c'est donc bien au total à l'analyse des relations entre ordre politique et ordre social que contribuent les articles de ce dossier. De ce point de vue, non seulement la différenciation du champ politique se révèle être l'un des garants de l'ordre social, mais ses reconfigurations comme son autonomisation croissante sont parties prenantes des transformations plus générales des modes de domination ${ }^{31}$.

\footnotetext{
${ }^{28}$ Ivan Bruneau, Gilles Laferté, Julian Mischi, Nicolas Renahy (dir.), Mondes ruraux et classes sociales, Paris, Editions de l'EHESS, 2018.

${ }^{29}$ À rebours donc d'une tendance à la décontextualisation des cas observable dans la sociologie reposant sur de la statistique inférentielle et visant à identifier l'effet propre de variables. Sur ces questions : Andrew Abbott, Time Matters: On Theory and Method, Chicago, University of Chicago Press, 2001.

${ }^{30}$ Rémy Caveng, Fanny Darbus, François Denord, Delphine Serre, Sylvain Thine, « Des morales de classe ? Dispositions éthiques et positions sociales dans la France contemporaine », Actes de la recherche en sciences sociales, n²24, 2018, p. 76-101; Amélie Beaumont, Raphaël Challier, Guillaume Lejeune, «En bas à droite. Travail, visions du monde et prises de position politiques dans le quart en bas à droite de l'espace social », Politix, n $^{\circ}$ 22, 2018, p. 9-31.

${ }^{31}$ Pierre Bourdieu, «Les modes de domination », Actes de le Recherche en Sciences Sociales, ${ }^{\circ} 2-3,1976$, p. 122-132. Une piste serait d'approfondir, par analogie, l'étude des transformations des intermédiations entre l'espace social et d'autres champs proches du champ du pouvoir.
} 